\title{
Sampling to Detect Rare Diseases
}

\author{
By Preben Willeberg
}

Danish Slaughterhouses, Axelborg, Axeltorv 3, DK-1609 Copenhagen V, Denmark.

\begin{abstract}
Monitoring / surveillance and estimation / detection of specific diseases are common procedures used in national disease control in farmed animals. When the disease is assumed to be rare or even absent, such activities are especially important, e.g. in documenting a preferable zoosanitary status as part of trade agreements. The statistical and epidemiological aspects of designing such investigations and the difficulties in properly analysing and interpreting the data are far from trivial. This presentation will point to some of these problems and it will suggest ways and means of minimising some of the difficulties.

Screening for disease to estimate its prevalence, e.g. during the course of a control or eradication program, must be based on a valid sampling scheme. Examples of such will be given from recent field studies.

Documenting freedom from disease must similarly be based on sampling when the aim is to document a continued freedom from a disease never found in, or previously eradicated from, the population. It is important to realise, how-
\end{abstract}

ever, that proving initial absolute freedom from a new or exotic disease cannot be made from a representative sample of the "normal" population! Examples of the confusion created by not recognising this distinction will be given.

Another common problem in designing and interpreting farm animal disease sampling is the frequently neglected effect of clustering by herd and other units, which will be illustrated and discussed.

The validity of the diagnostic procedures used to characterise the individuals in the sample as either positive or negative are of similar importance. Estimation and optimisation of the test sensitivity and specificity are integral parts of performing a survey or maintaining a surveillance programme.

Various methods to improve test sensitivity and specificity will be mentioned, and the importance of being able to estimate these characteristics will be illustrated. Newer methods for these purposes have been introduced in the field, and these will be discussed. 